\title{
Rotational bosonic current in a quasi-condensate confined in an optical toroidal trap
}

\author{
Aranya B. Bhattacherjee $\dagger^{*}$, E. Courtade $\nmid$ and E. Arimondo $† \|$ \\ † INFM, Dipartimento di Fisica E. Fermi, Università di Pisa, Via Buonarroti 2, \\ I-56127 Pisa, Italy \\ * Department of Physics, A.R.S.D. College, University of Delhi (South Campus), \\ Dhaula Kuan, New Delhi-110021, India
}

\begin{abstract}
We investigate the possibility of inducing a bosonic current which is rotational $(\vec{\nabla} \times \vec{v} \neq \overrightarrow{0})$ in a pseudo 1D quasi-condensate confined in an optical toroidal trap. The stability of such a current is also analyzed using hydrodynamics approach. We find that such a current is uniform when the circular symmetry is preserved and energetically stable when the modes of elementary excitations are restricted to one dimension. This scheme allows to distinguish between a quasi and a true condensate by measuring the rotational spectra of the sample.
\end{abstract}

|| To whom correspondence should be addressed (arimondo@df.unipi.it) 


\section{Introduction}

Bose-Einstein condensates provide an important quantum system where one can observe many important phenomena such as superfluid-Mott insulator transition [1] and vortices [2, 3, 4, 5]. Recent advances in experimental techniques [6, 7] have stimulated interest in one-dimensional (1D) Bose gases. The 1D case leads to some interesting physics which does not occur in higher dimensions. The experimental feasibility of realizing a tightly confined (1D) trapped gas has been investigated theoretically using Bessel light beams [8]. It is well known that a true BEC cannot occur at any finite temperature in an interacting homogeneous Bose gas in 1D [9], due to long wavelength fluctuations; nor can it occur in the limit of zero temperature [10], due to quantum fluctuations. However, the presence of a trapping potential changes the density of states at low energies, and in the weakly-interacting limit a BEC may be formed [11]. Recently Petrov et al. discussed three different regimes of quantum degeneracy which can occur in a condensate confined in 1D trap 12]: BEC, quasi-condensate, and Tonks-Girardeau gas. In the weakly interacting limit a BEC exists, but as the interaction becomes stronger the mean field energy becomes important. When this energy becomes of the order of the energy level spacing of the trap, fluctuations again become significant. In this regime, the system forms a quasi-condensate, with local phase coherence, rather than the global coherence associated with a true condensate. The quasi-condensate density has the same smooth profile as a true condensate but the phase fluctuates in space and time. Phase fluctuations of the condensate are caused mainly by low energy collective excitations [12. Quasi-condensates in 3D have been observed experimentally in equilibrium [13, 14] and non-equilibrium [15. The fact that the velocity of a true condensate $\overrightarrow{v_{s}}(\vec{r}, t)=\hbar / m \vec{\nabla} \phi(\vec{r}, t)$ (with $\phi(\vec{r}, t)$ the phase of the condensate) leads to the important constraint namely irrotationality $\vec{\nabla} \times \overrightarrow{v_{s}}(\vec{r}, t)=0$. On the other hand, the normal component which is substantially higher in a quasi-condensate as compared to a true condensate is not constrained by the condition of irrotationality. Hence we take advantage of this property of the quasi-condensate and propose in this work the use of a $2 \mathrm{D}$ optical rotator to produce a rotational bosonic current $(\vec{\nabla} \times \vec{v} \neq 0$ where $\vec{v}=\overrightarrow{v_{s}}+\overrightarrow{v_{n}}$ and $\overrightarrow{v_{n}}$ is the velocity of the normal component) in a quasi condensate and study the conditions under which this current is stable. We show that using this scheme, it is possible to distinguish between quasi-condensate and true condensate. It is to be noted that such a current is not vortex. Such a kind of a rotator has been proposed for cold atoms based on the principle of transfer of angular momentum of the photons to the atoms [16] using a TEM $_{01}$ circularly polarized Laguerre-Gaussian beam. It has been experimentally demonstrated that a blue-detuned hollow Bessel laser beam formed by an axicon can be successfully used to trap laser cooled metastable xenon atoms [17]. Experimentally, cesium atoms have been trapped in an all blue-detuned stack of optical rings by interference of a standard gaussian beam with a counter-propagating hollow beam generated by a pair of axicon [18, 19]. We propose the use of such an experimental scheme with a $\mathrm{TEM}_{01}$ beam in order to realize the $2 \mathrm{D}$ optical rotator. 


\section{Description of the optical torus}

We will examine the motion of a condensate within an optical toroidal ring trap as experimentally realized in Ref. [19] and previously theoretically analyzed by Wright et al. 16] The potential created by the optical laser may be written as

$$
V(r)=\frac{1}{2} m \omega_{r}^{2}\left(r-r_{0}\right)^{2}+\frac{1}{2} m \omega_{z}^{2} z^{2}
$$

where $x^{2}+y^{2}=r^{2}$ and $r_{0}$ is the location of the potential minima and also the mean radius of the torus. $\omega_{z}\left(\omega_{r}\right)$ is the oscillation frequency along the $z(r)$ direction. Because we suppose the plane of the ring perpendicular to the direction of gravity $(z$ direction in our system), the atomic energies are not effected by gravity. The optical torus described by the potential of Eq. (11) is represented in Fig. 11.

The atomic motion within the toroidal trap includes also a rotational energy along the center of the torus, described by the following rotational Hamiltonian:

$$
H_{r}=-\frac{\hbar^{2}}{2 I} \frac{\delta^{2}}{\delta \theta^{2}}
$$

where the moment of inertia for an atom with mass $m$ is given by $I=m r_{0}^{2}$. If $p$ is the rotational quantum number, then the rotational hamiltonian defines an energy spectrum whose energy spacing is linked to the rotation frequency $\Omega$ for $\Delta p=1$ as

$$
\Omega=\frac{(2 p+1) \hbar}{2 m r_{0}^{2}}
$$

Note that the rotational frequency $\Omega$ depends only on the radius of the $\operatorname{ring} r_{0}$. In the radial direction, the ring radius is approximately the radius of the laser beam, which therefore should be chosen as small as possible for a tight confinement. Extending the experimental parameters of [18] to Rb atoms, with a beam radius of about $50 \mu \mathrm{m}$, potential height of $10 E_{R}$ and $\omega_{R} \sim 2 \pi \times 0.34 \mathrm{kHz}$, one can find $\omega_{r} \sim 2 \pi \times 0.06 \mathrm{kHz}$, $\omega_{z} \sim 2 \pi \times 6.7 \mathrm{kHz}$ and $\Omega \sim 2 \pi \times 0.024 \mathrm{~Hz}$ (for $p=0 \rightarrow p=1$ transition).

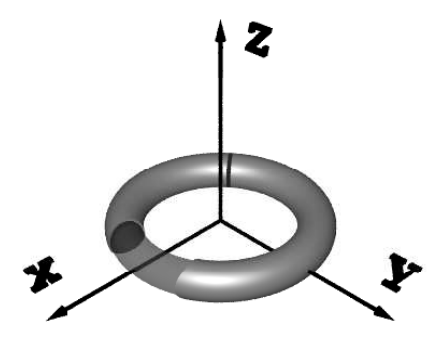

Figure 1. Schematic representation of the optical torus described by Eq. (11). This optical torus can be generated by the interference of a standard gaussian beam with a counter-propagating hollow beam generated by a pair of axicon [18, 19]. 
Moreover we will suppose, as in Ref. [16] that using for the laser field an high order circularly polarized Laguerre-Gaussian mode, an orbital angular momentum is transfered by the laser field to the atoms. Therefore the rotational motion of the condensate atoms at the frequency $\Omega$ can be excited by the laser field. Let us now systematically investigate the collective modes of a true condensate and that of a quasicondensate (in the presence of a rotational velocity field) in an optical torus.

\section{Collective modes of a true condensate in an optical torus}

For a true condensate the velocity is the gradient of a scalar, the velocity field is thus irrotational, unless the phase of the order parameter has a singularity (vortex). A simple description of the collective oscillations is provided by the irrotational hydrodynamics equations of superfluids

$$
\begin{aligned}
& \frac{\partial n_{s}}{\partial t}+\vec{\nabla} \cdot\left[n \overrightarrow{v_{s}}\right]=0 \\
& m \frac{\partial \overrightarrow{v_{s}}}{\partial t}+\vec{\nabla}\left[\frac{m v_{s}^{2}}{2}+V(\vec{r}, t)+n_{s} g_{3 d}\right]=0
\end{aligned}
$$

where $n_{s}(\vec{r}, t)$ is the superfluid spatial density, $v_{s}(\vec{r}, t)$ is the superfluid velocity field in the laboratory frame and $m$ is the mass of the atom. The parameter $g_{3 d}$ characterizes the strength of the inter-atomic interactions and is related to the $s$-wave scattering length $a_{s}$ for a 3D gas by $g_{3 d}=4 \pi \hbar^{2} a_{s} / m$. These equations are valid in the Thomas-Fermi limit, where the so-called quantum pressure term is neglected. The density can be written as $n_{s}=n_{0}+\delta n_{s}$, and $\overrightarrow{v_{s}}=\overrightarrow{v_{0}}+\delta \overrightarrow{v_{s}}$ where $n_{0}\left(\overrightarrow{v_{0}}\right)$ is the equilibrium density (velocity) and $\delta n_{s}\left(\delta \overrightarrow{v_{s}}\right)$ is a small perturbation of the density (velocity) from its equilibrium value. The collective oscillations can be derived by looking for a general time-dependent solutions of the form (see [20, 21])

$$
\begin{aligned}
& \delta n_{s}(\vec{r}, t)=\alpha_{0}+\alpha_{1}\left(x^{2}+y^{2}\right)+\alpha_{2} z^{2}+\alpha_{3} x y+\alpha_{4} x z+\alpha_{5} y z \\
& \delta \overrightarrow{v_{s}}(\vec{r}, t)=\vec{\nabla}\left(\beta_{0}+\beta_{1}\left(x^{2}+y^{2}\right)+\beta_{2} z^{2}+\beta_{3} x y+\beta_{4} x z+\beta_{5} y z\right)
\end{aligned}
$$

where $\alpha_{i}$ and $\beta_{i}$ are time dependents parameters to be determined. In the linear limit, one can look for solutions varying in time like $e^{-i \omega t}$. Linearizing Eqs. (4) and (5) and inserting $\delta n_{s}$ and $\delta \overrightarrow{v_{s}}$ and solving for the modes $\omega$ for $\omega_{x}=\omega_{y}=\omega_{r}$, one finds

$$
\omega_{ \pm}^{2}=\frac{3}{2}\left(\omega_{r}^{2}+\omega_{z}^{2}\right) \pm \frac{1}{2} \sqrt{9 \omega_{r}^{4}+9 \omega_{z}^{4}-10 \omega_{r}^{2} \omega_{z}^{2}}
$$

These modes describe the coupling between the center of mass motion along the radial and axial directions. Fig. 2 displays these modes as a function of the radial frequency which are seen to be stable. The frequencies are normalized with respect to the $\omega_{z}$ axial frequency. We now allow for the condition of tight confinement, i.e. the particles are constrained to move only along the path $x^{2}+y^{2}=r_{0}^{2}$. In the tight

confinement regime, at sufficiently low temperatures the radial motion of particles (the 
center of mass modes along $x$ and the $y$ direction) is essentially frozen if the radial frequency $\omega_{r}$ is much more than the mean-field interaction energy. Note that the tight confinement regime is not the $1 \mathrm{D}$ regime as discussed in [12. In our system, we still have the velocity component in both $x$ and $y$ directions. We prefer to call it as the pseudo 1D regime. Proceeding as before, we find in the tight confinement regime the two modes corresponding to center of mass motion along the $z$ direction and the scissoring modes $x z(y z)$ as $\omega^{2}=3 \omega_{z}^{2}$ and $\omega^{2}=\omega_{z}^{2}$ respectively. These low energy excitations frequencies are exactly the lowest mode of excitation found numerically in [22] which confirms the accuracy of the hydrodynamic approach.

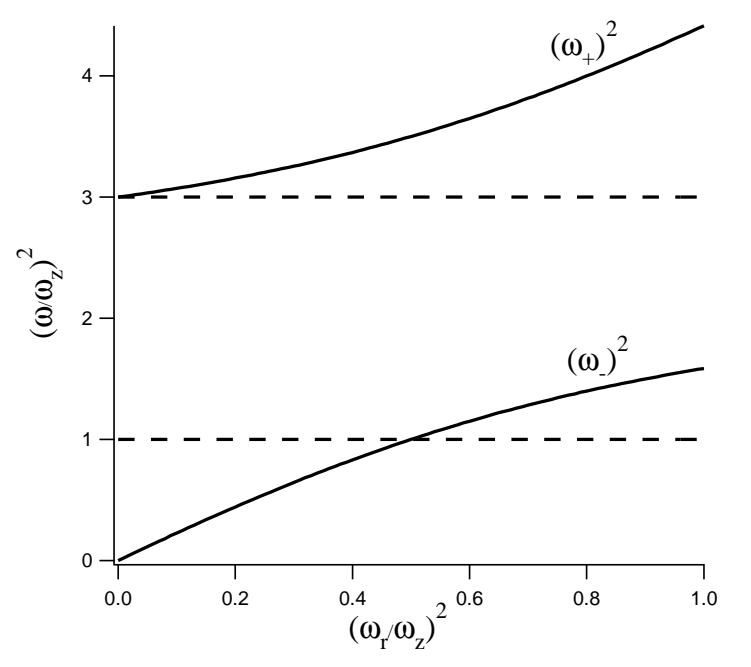

Figure 2. Mode frequencies of Eq. (8) as functions of the radial trap frequency $\omega_{r}$. The solid lines correspond to the modes of the true condensate in the optical torus. The dashed lines correspond to the modes of the condensate in the case of tight confinement in the optical torus. The positive values of the mode frequencies ensures stability.

\section{Collective modes of a quasi-condensate in an optical torus}

\subsection{Rotational velocity field of a quasi-condensate}

The special properties of superfluids are a consequence of their motions being constraint by the fact that the velocity $\left(\overrightarrow{v_{s}}(\vec{r}, t)\right)$ of the condensate is proportional to the gradient of the phase $(\phi(\vec{r}, t))$ of the wave function that is $\overrightarrow{v_{s}}(\vec{r}, t)=\hbar / m \vec{\nabla} \phi(\vec{r}, t)$. We see directly from this definition that $\overrightarrow{v_{s}}(\vec{r}, t)$ satisfies two important constraints, namely the condition of irrotationality $\vec{\nabla} \times \overrightarrow{v_{s}}(\vec{r}, t)=0$ and the Onsager-Feynman quantization condition $\oint \overrightarrow{v_{s}}(\vec{r}, t) \cdot d \vec{l}=n h / m(n=0, \pm 1, \pm 2 \ldots)$. Obviously, in a simply connected geometry with condensate wave function finite everywhere, the condition of irrotationality implies the Onsager-Feynman quantization condition with $n=0$, but for 
more general cases involving vortices or in a torus (multiply connected geometry), $n$ can be non zero.

In stable or metastable equilibrium the total bosonic current will have contribution from both the normal as well as the superfluid component (Landau's two fluid hypothesis). Now if by some mechanism, the condensate in the torus with mean radius $r_{0}$ is made to rotate with an angular velocity $\Omega$, then the linear velocity of the normal component will be simply $\Omega r_{0}$ while the linear velocity of the superfluid component will be constrained by the quantization condition and in general can not be equal to $\Omega r_{0}$. In fact, a simple statistical mechanical argument shows that the lowest free energy is obtained when $n$ takes the value closest to $\Omega / \Omega_{c}$ where $\Omega_{c}=\hbar / m r_{0}^{2}$. For $\Omega / \Omega_{c} \ll 1, n$ is equal to zero and consequently the superfluid component no longer contributes to the circulating current. At larger values of $\Omega\left(>\Omega_{c} / 2\right)$, the superfluid will contribute to the total angular momentum an amount $\sim n \Omega_{c}$. An inspection of Eq. (3) for $p=0 \rightarrow p=1$ transition together with the definition of $\Omega_{c}$, we find that $\Omega / \Omega_{c}=0.5$ irrespective of the radius of the $2 \mathrm{D}$ optical rotator. Consequently, in a true condensate, where the normal component is extremely low, the total bosonic current is almost zero for $\Omega / \Omega_{c} \ll 1$. Based on the above discussion, we argue that in a quasi-condensate, where the normal component is substantially higher than a true condensate, for $\Omega / \Omega_{c} \ll 1$, the normal component will contribute significantly to the bosonic current, which one could detect. Thus using this proposal, it could be possible to distinguish between a quasi-condensate and a true one.

We propose that the theoretical scheme introduced in [16] may be used to transfer orbital angular momentum of the photon to a cloud of ultra-cold atoms in a quasicondensate state. A velocity field created in such a manner is rotational. This velocity field can be visualized by introducing a vector potential $\vec{A}$ in the quasi-condensate total velocity $\vec{v}=\overrightarrow{v_{s}}+\overrightarrow{v_{n}}$

$$
\vec{v}(\vec{r})=\frac{\hbar}{m}[\nabla \phi(\vec{r})+\vec{A}]
$$

where the vector potential $\vec{A}$ is associated with the rotational component of the velocity

$$
\vec{A}=\frac{m}{\hbar} \vec{\Omega} \times \vec{r}
$$

with $\vec{\Omega}=\Omega \widehat{k} . \Omega$ is the rotational angular velocity of the sample. The corresponding rotational velocity field which is actually the velocity of the normal component

$$
\vec{v}_{r o t}=\vec{v}_{n}=\Omega(-y \widehat{i}+x \widehat{j})
$$

with $\vec{\nabla} \times \vec{v}_{n}=2 \vec{\Omega}$. The angular velocity of the sample is easily adjusted by the mean ring radius $r_{0}$ (see Eq. (3) $)$. 


\subsection{Stability conditions for stable persistent current}

Let us now analyze the condition for existence of a steady velocity field $\vec{v}_{n}$ of the form of Eq. (11) in a quasi-condensate for a tight confinement in the presence of a weak perturbation (a slightly distorted potential). The perturbed potential is written as

$$
V(r)=\frac{1}{2} m \omega_{r}^{2}\left(r-r_{0}\right)^{2}+\frac{1}{2} m \omega_{z}^{2} z^{2}+\frac{1}{2} m\left(\delta \omega_{x}\right)^{2} x^{2}+\frac{1}{2} m\left(\delta \omega_{y}\right)^{2} y^{2}
$$

where $\delta \omega_{x}$ and $\delta \omega_{y}$ are small external perturbations in the frequencies along $x$ and $y$ direction. In the presence of the perturbation, the irrotational component of the velocity field is now also included which is written as $\vec{v}_{\text {irrot }}=\vec{v}_{s}=\alpha \vec{\nabla}(x y)$. Therefore the total velocity field is

$$
\vec{v}(\vec{r})=(\alpha-\Omega) y \widehat{i}+(\alpha+\Omega) x \widehat{j}
$$

The macroscopic description of modes with circulation is provided by the equations of two component rotational hydrodynamics [21, 23]

$$
m \frac{\partial \vec{v}}{\partial t}+\vec{\nabla}\left[\frac{m v^{2}}{2}+V(\vec{r}, t)+g_{3 d} n_{s}+2 g_{3 d} n_{n}\right]=m \vec{v} \times(\vec{\nabla} \times \vec{v})
$$

In the following, we will ignore fluctuations in the density of the normal component $\left(n_{n}\right)$ and also neglect damping due to interaction between superfluid and normal component. The continuity equation leads to the following expression for $\alpha$

$$
\alpha=\frac{\Omega\left(\left(\delta \omega_{y}\right)^{2}-\left(\delta \omega_{x}\right)\right)^{2}}{2 \Omega^{2}-\left(\left(\delta \omega_{y}\right)^{2}+\left(\delta \omega_{x}\right)^{2}\right)}
$$

Thus we see from Eq. (13) that the irrotational component of the velocity field which comes into picture as a result of the perturbation destroys the bosonic current with uniform angular velocity $\Omega$. From Eq. (15) we infer that for such a uniform current to exist, we must have $\left(\delta \omega_{x}\right)^{2}=\left(\delta \omega_{y}\right)^{2}$. The conservation of circular symmetry guarantees a uniform current in the quasi-condensate. In the presence of a weak, static, asymmetric perturbation, angular momentum is not conserved. In a rotating quasi-condensate, scattering of particles against such an asymmetric perturbation will ultimately bring the fluid to rest. Having found that circular symmetry as a necessary criterion for a uniform current to exist, we now proceed to calculate the collective modes in the nonequilibrium state. Collective modes in the presence of a rotational velocity field for a trap with circular symmetry can be derived as in section 3 by looking for time-dependant solutions for $\delta n$ and $\delta \vec{v}$. We now allow for tight confinement and assume that we still have motion in $x z(y z)$ planes and also center of mass motion along the $z$ direction. The

density and velocity fluctuations are now proportional to $z^{2}, x z, y z$. These modes from the usual hydrodynamic approach are found to be

$$
\omega^{2}=3 \omega_{z}^{2}
$$

and roots of the equation

$$
\omega^{3}+\Omega \omega^{2}-\left(\Omega^{2}+\omega_{z}^{2}\right) \omega-\Omega\left(\Omega^{2}+\omega_{z}^{2}\right)=0
$$


The modes calculated from of Eq. (16) and Eq. (17) are plotted in Fig. 3, Negative frequencies of excitations are taken as signature of energetic instability which is a result of the absence of thermodynamic equilibrium [24]. The mode corresponding to the center of mass motion along the $z$ direction from Eq. (16) (dashed line in Fig. 31) is always positive and constant. This non dependence on $\Omega$ is a consequence of the absence of excitations in the $x y$ plane due to tight confinement. We find that for a non-zero value of $\Omega$, one of the scissoring modes in the $x z(y z)$ planes of Eq. (17) (lower curve in Fig. 3) is always negative. This signals energetic instability because the magnitude of the smallest root is always equal to the angular velocity $\Omega$. This is equivalent to the Landau criterion of super-fluid stability [25, 26] with respect to proliferation of elementary excitations i.e. the bosonic current becomes unstable when the frequency of the lowest energy excitation becomes equal or more than the angular velocity. A similar result is found for a Bose gas in a toroidal container using Bogoliugov approximation 27. Thus to have an energetically stable current we need to freeze the motion in $x z$ and $y z$ planes. This means that we restrict the modes of the elementary excitations to only the $z$ direction whose frequency is given by Eq. (16).

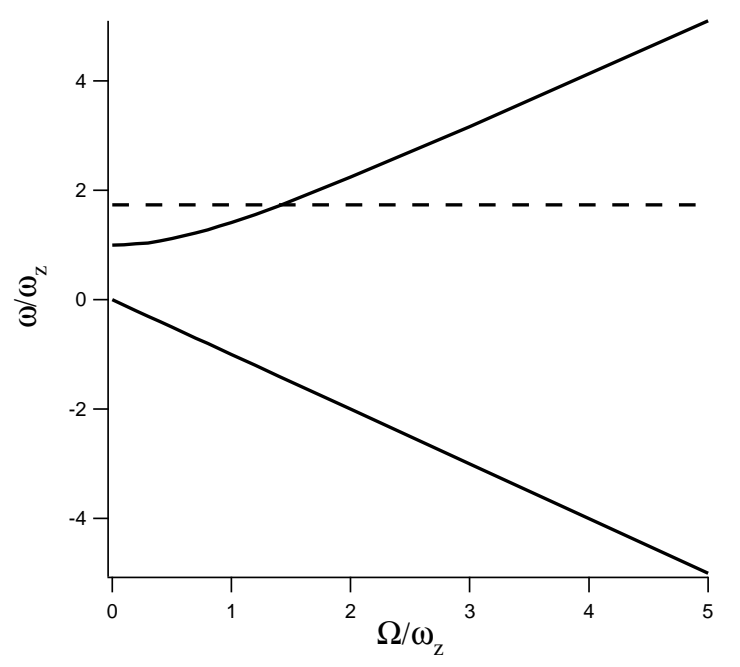

Figure 3. Calculated modes frequencies as functions of the angular frequency $\Omega$. The dashed line corresponds to the center of mass frequency along the $z$ direction from Eq. (16). The other two solid line curves correspond to the scissoring modes in the $x z(y z)$ planes of Eq. (17). The negative frequencies of the lower curve are signature of instability (see text).

Let us now look into the possible experimental realization of such a pseudo quasicondensate in our proposed scheme. The mean field interaction frequency $g_{3 d} N / \hbar 2 \pi r_{0}^{3}$ for $N=10^{4}$ and $a_{s}=5 \mathrm{~nm}$ (in the case of $\mathrm{Rb}$ ) is found to be $\sim 2 \pi \times 0.5 \mathrm{~Hz}$ much less than the radial trap frequency $(\sim 2 \pi \times 60 \mathrm{~Hz}$, see section 21) which implies that we are indeed in the pseudo 1D regime. Following [12, one can introduce a dimensionless quantity $\alpha=4 \pi a_{s} / l$, where $l=\sqrt{\hbar / m \omega_{z}}$ is the amplitude of axial zero point oscillations. 
The regime of a weakly interacting gas corresponds to $\alpha \ll 1$. In this regime, the condensate is in the Thomas-Fermi regime if the number of particles $N>\alpha^{-1}$. The decrease of temperature to below the degeneracy temperature $k_{B} T_{d}=N \hbar \omega_{z}$ leads to the appearance of a quasi-condensate which at $T<T_{c r}$ turns to a true condensate. The

critical temperature $T_{c r}$ is determined by $k_{B} T_{c r}=\hbar \omega_{z}\left(32 N / 9 \alpha^{2}\right)^{1 / 3}$. For $N \sim 10^{4}$ and using the values of the trap parameters given earlier (see section 2), we find $T_{d}=3 \mathrm{mK}$ and $T_{c r}=17 \mu \mathrm{K}$. Working between these two temperature limit and having $\omega_{r}>g_{3 d} N / \hbar 2 \pi r_{0}^{3}$, one can thus be in the pseudo $1 \mathrm{D}$ quasi-condensate regime. This scheme allows us to distinguish between a quasi-condensate and a true condensate by simply measuring the rotational spectrum of the sample. The rotational spectrum of the sample can be measured by using two consecutive Bragg pulses probing the momentum distribution in a trapped Bose gas at low temperature [28, 29]. A stimulated Raman transition involves absorption of a photon from one beam followed by stimulated emission into the other; if the system is in a quasi-condensate regime then this process will transfer an orbital angular momentum off to the atom, but no linear momentum in the axial or radial directions.

\section{Conclusion}

In conclusion, we have proposed the use of a new type of optical toroidal trap to induce a uniform rotational bosonic current (which is not a vortex) in a quasi-condensate. The principle of producing such a current of rotational is based on the transfer of angular momentum of the photon to the atoms of the normal component. The angular velocity induced by the proposed 2D optical rotator is exactly half the critical angular velocity required by the superfluid component to contribute to the total bosonic current. Hence, the given optical rotator is unable to transfer any angular momentum to the atoms in the superfluid component. We are thus able to distinguish between a quasi-condensate and a true condensate by measuring the rotational spectrum of the sample by two-photon Bragg spectroscopy. Our analysis indicates that such a rotational current is uniform when the circular symmetry of the ring trap is conserved and energetically stable when the elementary excitations are completely frozen in the transverse direction ( $x y$ plane).

\section{Acknowledgments}

Fruitful discussions with I. Carusotto and A. Recati, and a careful check of the manuscript by C. Menotti are acknowledged. This research was supported by the Abdus Salam International Centre for Theoretical Physics, Trieste, Italy under the ICTP-TRIL fellowship scheme and the Sezione A of INFM-Italy through a PAIS Project, by the MIUR-Italy through a COFIN Project, and by the European Commission through the Cold Quantum-Gases Network, contract HPRN-CT-2000-00125. 


\section{References}

[1] M. Greiner, O. Mandel, T. Esslinger, T. W. Hansch, and I. Bloch, Nature 415, 39 (2002).

[2] M. R. Matthews, B. P. Anderson, P. C. Halijan, D. S. Hall, C. E. Wieman, and E. A. Cornell, Phys. Rev. Lett. 83, 2498 (1999).

[3] K. W. Madison, F. Chevy, W. Wohlleben, and J. Dalibard, Phys. Rev. Lett. 84, 806 (2000).

[4] K. W. Madison, F. Chevy, W. Wohlleben, and J. Dalibard, J. Mod. Opt. 47, 2715 (2000).

[5] F. Chevy, K. W. Madison, and J. Dalibard, Phys. Rev. Lett. 85, 2223 (2000).

[6] A. Gorlitz, J. M. Vogels, A. E. Leanhardt, C. Raman, T. L. Gustavson, J. R. Abo-Shaeer, A. P. Chikkatur, S. Gupta, S. Inouye, T. Rosenband, and W. Ketterle, Phys. Rev. Lett. 87, 130402 (2001).

[7] F. Schreck, L. Khaykovich, K. L. Corwin, G. Ferrari, T. Bourdel, J. Cubizolles, and C. Salomon, Phys. Rev. Lett. 87, 080403 (2001).

[8] J. Arlt, K. Dholakia, J. Soneson and E.M. Wright, Phys. Rev. A 63, 063602 (2001).

[9] P. C. Hohenberg, Phys. Rev. 158, 383 (1967).

[10] L. Pitaevskii and S. Stringari, J. Low Temp. Phys. 85, 377 (1991).

[11] W. Ketterle and N. Van Druten, Phys. Rev. A 54, 656 (1996).

[12] D. S. Petrov, G. V. Shlyapnikov, and J. Walraven, Phys. Rev. Lett. 85, 3745 (2000).

[13] S. Dettmer, D. Hellweg, P. Ryytty, J. J. Arlt, W. Ertmer, K. Sengstock, D. S. Petrov, and G. V. Shlyapnikov, Phys. Rev. Lett. 87, 160406 (2001).

[14] D. Hellweg, S. Dettmer, P. Ryytty, J. J. Arlt, W. Ertmer, K. Sengstock, D. S. Petrov ,G. V. Shlyapnikov, H. Kreutzmann, L. Santos, and M. Lewenstein, Appl. Phys. B: Lasers Opt. 73, 781 (2001).

[15] I. Shvarchuck, Ch. Buggle, D. S. Petrov, K. Dieckmann, M. Zielonkowski, M. Kemmann, T. G. Tiecke, W. Von Klitzing, G. V. Shlyapnikov, and J. T. M. Walraven, Phys. Rev. Lett. 89, 270404 (2002).

[16] E. M. Wright, P. S. Jessen, and G. J. Lapeyere, Opt. Comm. 129, 423 (1996).

[17] S. Kulin, S. Aubin, S. Christe, B. Peker, S.L. Rolston and L.A. Orozco, J. Opt. B: Quantum Semiclass. Opt. 3353 (2001).

[18] B. Depret, P. Verkerk, and D. Hennequin, J. Phys. IV 12 (PR5), 137 (2002)-B. Depret, P. Verkerk, and D. Hennequin, Opt. Comm. 211, 31 (2002).

[19] P. Verkerk and D. Hennequin, physics/0306155 (2003).

[20] C. Menotti and S. Stringari, Phys. Rev. A 66, 043610 (2002).

[21] M. Cozzini and S. Stringari, Phys. Rev. A 67, 041602 (2003).

[22] L. Salasnich, A. Parola, and L. Reatto, Phys. Rev. A 59, 2990 (1999).

[23] M. Griffin, Mod. Phys. Lett. B 14, Suppl. Issue, 65 (2000).

[24] A. Recati, F. Zambelli and S. Stringari, Phys. Rev. Lett. 86, 377 (2001).

[25] J. Javanainen, S. M. Paik, and S. M. Yoo, Phys. Rev. A 58, 580 (1997).

[26] J. Javanainen and Yi Zheng, Phys. Rev. A 63, 063610 (2001).

[27] D. S. Rokhsar, cond-math/9709212 (1997).

[28] J. Stenger, S. Inouye, A. P. Chikkatur, D. M. Stamper-Kurn, D. E. Pritchard, and W. Ketterle, Phys. Rev. Lett. 82, 4569 (1999).

[29] A. Brunello, F. Dalfovo, L. Pitaevskii, and S. Stringari, Phys. Rev. Lett. 85, 4422 (2000). 